\title{
Positive Impact of Computer Conferencing in Distance Education
}

\author{
Gbenga Michael Adeyeye
}

$\mathrm{PhD}$, College of Education, Department of Educational Foundation, University of South Africa

\begin{abstract}
A number of studies have examined the dynamics and style of communication adopted in computer conferencing in distance education (Mason, 1993). Computer conferencing by higher education institutions offering distance education courses has expanded rapidly since 1987. Lately computer conferencing has developed in fame as a vehicle of distance education. While protecting the opportunity related with asynchronous communication and giving wide access to low-end innovation users, computer conferencing enables students and instructors to take part in group learning and associate 'many-to-many' (Harasim, 1989). Much has already been written on the subject of computer conferencing in support of educational processes, in particular distance education (DE). Using unstructured interviews and observations at adult students' homes or worksites, the study investigated adult student perspectives of distance study by computer conferencing. The focal finding of this investigation was that computer conferencing can bolster a scope of learning situations, from teacher-led symposium to symposium to a student-centered community workshop. In addition, adults actively engaged in social relationships outside their distance studies which sustain their educational pursuits. The students provided insights into aspects of the on-line environment: as synchronicity, interactivity, textual communications, and collaboration.
\end{abstract}

Keywords: positive, impact, computer conferencing, distance education

\section{Introduction}

A number of studies have examined the dynamics and style of communication adopted in computer conferencing in distance education (Garrison, D. R., Anderson, T., \& Archer, W. 2001). Computer conferencing by higher education institutions offering distance education courses has expanded rapidly since 1987. Lately computer conferencing has developed in fame as a vehicle of distance education. While protecting the opportunity related with asynchronous communication and giving wide access to low-end innovation users, computer conferencing enables students and instructors to take part in group learning and associate 'many-to-many' (Harasim, 1989). Much has already been written on the subject of computer conferencing in support of educational processes, in particular distance education (DE). Naughton, N., \& Redfern, S. (2002) link the collaborative potential of computer conferencing to constructivist teaching and learning, he also claim that the new technologies have contributed to a movement away from a traditional objectivist paradigm that views education as the transmission of information from teacher to student, to one that views knowledge as a personally constructed world view. Naughton, N., \& Redfern, asserts that the collaborative potential of computer-mediated communication makes it an ideal tool for constructivist teaching. He proposes that distance educators can and should take advantage of this potential and encourage students to make their own meanings through collaboration with others: 'Through the process of articulating covert processes and strategies, learners are able to build new and modify existing knowledge structures. (Bonk, C. J., \& King, K. S. 2012). Notable researchers on computer conferencing assert that the asynchronous group interaction of the medium demands a shift in the teacher's role from information presenter to facilitator of discussion (McCabe, M. F. 1998)

\section{ONLINE EDUCATION}

The methodologies applied in first-and second-generation distance education frameworks (separately dependent on correspondence and multimedia) were and are focused to a great extent on the generation and conveyance of learning material to the learning network. Correspondence with the students (in a bi-directional sense) is kept to a minimum and correspondence among students is essentially inexistent. In this line, Distance Education is to a great extent decreased to 
a matter of covering geological gap (an issue that can be dealt with by viable techniques for the presentation and distribution of learning material). Therefore, learning is never again treated as a social procedure in which need is given to educator to student and communication between students, but instead as an only individual interest. The classes taking part in this type of Distance Education are virtual just in the feeling of being spread over a wide zone and thoroughly come up short on the socio-subjective component common of customary tutoring (Taylor, J. C. 1995).

The possibility that learning should keep on being a social procedure even at a distance was the Spring board for the improvement of third-generation distance education frameworks. In Distance Education system, third-generation frameworks are likewise called online training frameworks since a large portion of the learning procedure happens on PC systems. Here members cooperate with each other inside a veritable learning network in which people can defeat their seclusion and reinforce their association with the gathering. These strategies loan themselves especially well to grown-up instruction, a setting where the sharing of individual experience about the subject being contemplated can assume a crucial job in aggregate advancement (Benigno, V., \& Trentin, G. 2000). Moreover, to encourage association between members, numerous community learning techniques tried in on location instruction can be used in online training.

The requirement for an exceedingly organized communication environment in request to actualize the methodology described earlier , the computer network environment utilized must have the capacity to oversee "virtual classrooms" in which every one of the members (or a set thereof) may "meet" and spotlight all the more intently on different subjects canvassed in the course. The point is to allow, where essential, either the foundation of various work/contemplate bunches working in parallel or further dialog on finished course modules, and to do as such without upsetting the tutor's timetable of exercises; as it were, those keen on a specific action can stay with it while the others continue as indicated by the arranged calendar. Computer conferencing frameworks are regularly utilized for taking care of online instruction exercises as they allow the production of disseminated learning conditions that beat existence restrictions.

Interaction through computer conferencing frameworks gives new chances to overseeing and taking an interest in the learning procedure that were obscure in traditional distance learning and traditional on location courses.

Computer conferencing, and $\mathrm{CMC}$ as a rule, not just offer asynchronous many-to-numerous communication, they additionally make broad utilization of interaction through the written word (Mason, 1993). The style of these exchanges is to a great degree casual and is frequently alluded to in the literature as "say-writing". Interacting on a par with individual members creates various advantages for the learning procedure, particularly when conducted in writing. Planning and communicating one's very own thoughts, together with contemplating and responding to those of others, are important cognitive capacities. Moreover, active participation prompts an amazingly rich learning circumstance, enabling every member to analyze the subject being examined or a specific issue from different perspectives, i.e. those of other participant (Harasim, 1989).

Another intriguing element of computer conferencing is its adaptability in favoring and supporting collaborative learning exercises. It ought to be recollected, in any case, that planning and directing an online course dependent on CMC includes receiving a progression of techniques and methodologies that vary generously from those run of the mill of traditional distance education and on location training. Before embarking on the structure of an online training venture, it is thusly important to be completely mindful of the attributes of the picked telematics frameworks and of the correspondence elements they will in general empower in relational exchange between members.

\section{WEB CONFERENCING}

Can be explained as the environments based on the WWW standard which can be accessed using any of the most common web-browsers such as Netscape or Explorer. In order to understand the kind of problem that Web-conferencing seeks to tackle, a brief premise is needed. Systems which are purposely designed for computer conferencing are generally based on a client-server architecture: at one end is the network server on which the system is managed, and at the other end is a specific software application (the client) installed on the user site that can communicate with the server from a distance. These applications are usually specific to one particular environment and if the conferencing system is changed, it becomes necessary to use a different client application. Having to use different clients for different network services has been a recognised problem for some time, with the result that the most popular web-browsers now offer sub-environments for e-mail and accessing News services together with their normal navigation functions. 
In the specific case of computer conferencing, many producers have tried to solve the problem of specialised clients by adapting their systems to the WWW world. This has often led to hybrid environments that have only some of the functions contained in the original computer conferencing system - which, however, can still be reached if desired through the related client. As a consequence, computer conferencing environments that can be accessed via the WWW can be grouped into two distinct categories: Web-Conferencing Systems and Non-Web-Conferencing Systems (Woolley, 1996). The former are purposely designed for the WWW standard while the latter can be viewed through the WWW environment but are based on other standards.

Although great strides forward have been taken in the development of new Web-Conferencing systems, for the time being these do not offer the same wealth of functions as the most sophisticated computer conferencing systems. This is not due, of course, to the producers' shortcomings but to the limitations imposed by the WWW standard. According to an analysis published on the network by David Woolley (1996) there are also other problems that currently make these systems uncompetitive with computer conferencing systems. One of these is related to performance: when working on the Web, delays arise in navigation (moving from one site to another) and from the way Web pages come up on screen. Furthermore, the text editing capabilities of Web-based environments are decidedly poor and not at all comparable with those of the most popular computer conferencing environments. It should be stressed, however, that evolution in both the Hypertext Transfer Protocol Secure and the Hypertext Markup Language could bring improvements in performance and in user interfaces. Nevertheless, experts claim that in order to solve these problems completely, the HTML approach has to be totally bypassed and interfaces should be written directly in other languages (e.g. Java).

\section{IMPORTANT ASPECT OF ONLINE COURSE}

In online courses to structure communication to suit educational activities and the course's modular structure. Each online course module involves a series of activities based on a specific educational strategy which must be supported by wellmanaged communication between participants. Hence the need for a communication environment that can easily be molded, even while the course is underway, in accordance with the various types of interaction between students or with events that could not be foreseen in the planning stage HTTP: Hypertext Transfer Protocol Secure used in browser-server communication. HTML: Hypertext Markup Language: language used for programming Web pages.

The communication dynamics typical of online courses are varied and complex. They are strictly linked to course organization, the scheduling of activities (sometimes in a sequence, sometimes in parallel), and the existence of collaborative work environments and, last but not least, the teaching approaches adopted. All this translates into a series of precise functions which become the requirements of the computer conferencing system. We have seen that many systems tend to be open, in that they allow anyone with a web browser to gain access to the various conferences. In this respect, it has been noted that the choice between restricting the environment to selected users or keeping it open to everyone depends on the aims to be pursued. A first question could therefore be what product to choose if one intends to use a computer conferencing environment within an online course: a system that is accessible through a specific client or one that can be reached through any web-browser. By their nature, online courses address a limited number of participants. So the point is to fully exploit the system's ability to manage fairly small but varied groups and sub-groups, and grant them the utmost privacy as well. In online courses, much of the start-up period is dedicated to socialising and creating an atmosphere of conviviality in which individuals feel free to express themselves and are confident that they will only be heard by their tutors and peers. We should not overlook the fact that it takes a while to get used to the idea of writing things that will be memorized and will remain visible to others.

What's more, it should be remembered that computer conferencing systems often come with a "history" function, i.e. a record of the people who have read a given message. It might therefore make some uneasy to know that their messages have been read by third parties other than their peers or tutors, people with whom a certain relationship has already been established. Managers of online courses do not have to concern themselves so much with publicizing what happens inside the course, but rather with safeguarding the privacy of their virtual class. Going back to the above question, the problem of how to access the system (whether through a specialized client or a web-browser) becomes secondary to the choice of a suitable computer conferencing system that will provide the functions discussed above, namely:

- User customization of the desktop;

- An off-line reader, particularly for users connecting through modems; 
- Access management through a reliable, flexible mechanism for defining user

- permissions;

- Automatic storage and statistics of user activities;

- $\quad$ Advanced word-processing functions, i.e. not just standard ASCII text but also some standard editing formats (underlining, bold type, fonts, colour, etc.).

However, considering that for the time being the potential of computer conferencing systems can only be exploited to the full through specific clients, it remains advisable to conduct online courses in this way. Let's conclude this paper with a final reflection on text-based communication, which is typical of computer conferencing systems. Deferred text-based communication has proved to be an effective and relatively inexpensive way of managing and participating in thirdgeneration distance courses. However, just as many educational projects based on audio-video interaction have required the inclusion of asynchronous communication, online courses often call for complementary face-to-face events. These may even be carried out virtually, for instance using desktop conferencing (Trentin \& Benigno, 1997). Hence, many upcoming studies and pilot projects concerning the methodologies for applying communication technology to online education will have to focus on striking a balance between these communication modes, which are quite different but at the same time complementary.

\section{METHOD}

This study was undergone to encourage the utilization of modern computing to encourage distance education and investigate adult student perspectives of distance study by computer conferencing, qualitative methods using unstructured interviews and observations at adult students' homes or worksites was utilized to capture the impact of computer conferencing on distance education.

\section{FINDINGS}

In light of interview questions, the respondents depicted how computer conferencing has been of tremendous assistance to their studies. The exploration performed in this studies use computer conferencing as a mechanism for the conveyance of material to distance learners, and as a methods for encouraging communication among students and their tutors. The focal finding of this investigation was that computer conferencing can bolster a scope of learning situations, from teacherled symposium to symposium to a student-centered community workshop. Students additionally noticed that conferencing refered to the advantages as being snappy reactions to questions and a decreased sentiment of disconnection on account of the expanded capacity to speak with their friends and tutors. Different talks of the advantages and disadvantages of computer conferencing as it applies to distance learning can be found somewhere else. (Rimmershaw, 1999). This investigation likewise demonstrates that computer conferencing has the capability of turning into a communication/problemsolving medium that offers an authentic method to legitimately challenge the present quick float toward an impasse brought about by an excessive amount of data, too brief period to process it, and too little ability inside individuals alone to interrelate and assess data regardless of whether prepared.

\section{REFERENCES}

[1] Benigno, V., \& Trentin, G. (2000). The evaluation of online courses. Journal of computer assisted learning, 16(3), 259-270.

[2] Woolley, D. R. (1996). Conferencing on the Web. World Wide Web. Net Publishing, reprinted at.

[3] Garrison, D. R., Anderson, T., \& Archer, W. (2001). Critical thinking, cognitive presence, and computer conferencing in distance education. American Journal of distance education, 15(1), 7-23.

[4] Harasim, L. M. (1989). Online education: An environment for collaboration and intellectual amplification. Educational Evaluation Centre, Ontario Institute for Studies in Education.

[5] McCabe, M. F. (1998). Lessons from the field: Computer conferencing in higher education. Journal of Information Technology for Teacher Education, 7(1), 71-87.

[6] Naughton, N., \& Redfern, S. (2002). Collaborative virtual environments to support communication and community in internet-based distance education.

[7] Rimmershaw, R. (1999). Using conferencing to support a culture of collaborative study. Journal of Computer Assisted Learning, 15(3), 189-200. 
[8] Taylor, J. C. (1995). Distance education technologies: The fourth generation. Australian Journal of Educational Technology, 11, 1-7.

[9] Trentin, G., \& Benigno, V. (1997). Multimedia Conferencing in Education: Methodological and Organizational Considerations. Educational Technology, 37(5), 32-39. 